\title{
Synthesis, Biological Evaluation and Structure-Activity Relationships of Diflapolin Analogues as Dual sEH/FLAP Inhibitors
}

Lisa Vieider, ${ }^{\dagger, \#}$ Erik Romp, ${ }^{\ddagger, \nabla}$ Veronika Temml, ${ }^{\S,} \bigcirc$ Jana Fischer, ${ }^{\ddagger, \nabla}$ Christian Kretzer, ${ }^{\ddagger}, \nabla$ Martin Schoenthaler, ${ }^{\dagger, \#}$ Abdulla Taha, ${ }^{\ddagger, \nabla}$ Victor Hernández-Olmos, ${ }^{\perp, \mathbb{I}}$ Sonja Sturm, ${ }^{\S, \bigcirc}$

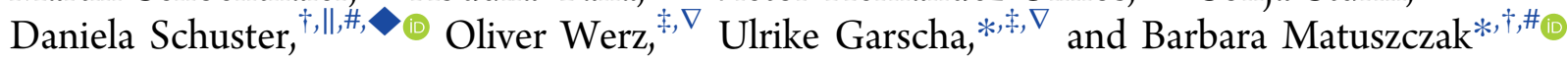

${ }^{\dagger}$ Institute of Pharmacy, Department of Pharmaceutical Chemistry, Center for Chemistry and Biomedicine, University of Innsbruck, Innrain 80-82, A-6020 Innsbruck, Austria

${ }^{\ddagger}$ Chair of Pharmaceutical/Medicinal Chemistry, Friedrich-Schiller University Jena, Philosophenweg 14, D-07743 Jena, Germany

${ }^{\S}$ Institute of Pharmacy, Department of Pharmacognosy, Center for Chemistry and Biomedicine, University of Innsbruck, Innrain 80-82, A-6020 Innsbruck, Austria

"Institute of Pharmacy, Department of Pharmaceutical and Medicinal Chemistry, Paracelsus Medical University Salzburg, Strubergasse 21, A-5020 Salzburg, Austria

${ }^{\perp}$ Fraunhofer Institute for Molecular Biology and Applied Ecology IME, Branch for Translational Medicine and Pharmacology TMP, Theodor-Stern-Kai 7, 60596 Frankfurt am Main, Germany

Supporting Information

ABSTRACT: A series of derivatives of the potent dual soluble epoxide hydrolase $(\mathrm{sEH}) / 5$-lipoxygenase-activating protein (FLAP) inhibitor diflapolin was designed, synthesized, and characterized by ${ }^{1} \mathrm{H} \mathrm{NMR}$, ${ }^{13} \mathrm{C} \mathrm{NMR}$, and elemental analysis. These novel compounds were biologically evaluated for their inhibitory activity against $\mathrm{sEH}$ and FLAP. Molecular modeling tools were applied to analyze structureactivity relationships (SAR) on both targets. Results show that even small modifications on the lead compound diflapolin markedly influence the inhibitory potential, especially on FLAP, suggesting very narrow SAR.

KEYWORDS: Soluble epoxide hydrolase (sEH), 5-Lipoxygenase activating protein (FLAP), Arachidonic acid cascade, Diflapolin, Inflammation

\begin{abstract}
A wide range of chronic pathologies (e.g., rheumatoid arthritis, hashimoto thyroiditis, and asthma bronchiale) is related to unresolved or misdirected inflammatory processes. Bioactive lipid mediators deriving from polyunsaturated fatty acids play central roles in inflammation on the one hand but also in resolution of inflammatory events. ${ }^{1}$ Upon cell stimulation, cytosolic phospholipase $\mathrm{A}_{2} \quad\left(\mathrm{cPLA}_{2}\right)$ releases arachidonic acid (AA) from phospholipids of cellular membranes, where specific oxygenases such as cyclooxygenases (COX)-1/2, 5-, 12-, and 15-lipoxygenases (LO) as well as cytochrome (CYP) P450 enzymes convert AA toward proinflammatory, pro-resolving as well as anti-inflammatory lipid mediators. ${ }^{2,3}$ COX inhibitors are still the standard treatment for acute inflammatory conditions and have been intensively investigated over the last decades. However, in long-term use, they cause severe gastrointestinal and cardiovascular side effects. ${ }^{4,5} \mathrm{CYP}$ and $\mathrm{LO}$ metabolic routes attracted attention to develop new anti-inflammatory drugs with less deleterious side effects. Moreover, it is well-known that crosstalk between the branches of AA cascade occurs. Thus, selective inhibition of one of the three pathways may cause a shunt toward the other two. ${ }^{2,6}$ The current strategy to combat chronic inflammation is
\end{abstract}

often the administration of a drug cocktail, sometimes coformulated in one tablet to improve patient compliance. Such a multicomponent therapy can suffer from drug-drug interactions, unexpected pharmacokinetic properties, or changes in pharmacodynamics. Drugs that interfere with multiple targets might provide a solution for these problems. Therefore, current research focuses on the development of designed multiple ligands (DMLs)., ${ }^{7,8}$ Compared to multicomponent drugs, there is only one active agent interfering with more than one target, which lowers the risk of toxicity and drug-drug interactions and offers the possibility for a low dose treatment. $^{2,8,9}$

Recently, diflapolin was identified as new dual inhibitor interfering with both the CYP and 5-LO pathway within the AA cascade. ${ }^{10,11}$ On the one hand, diflapolin (1) (Figure 1) targets the soluble epoxide hydrolase $(\mathrm{sEH})$, which is responsible for the rapid degradation of CYP-produced

Received: September 11, 2018

Accepted: November 29, 2018

Published: November 29, 2018 


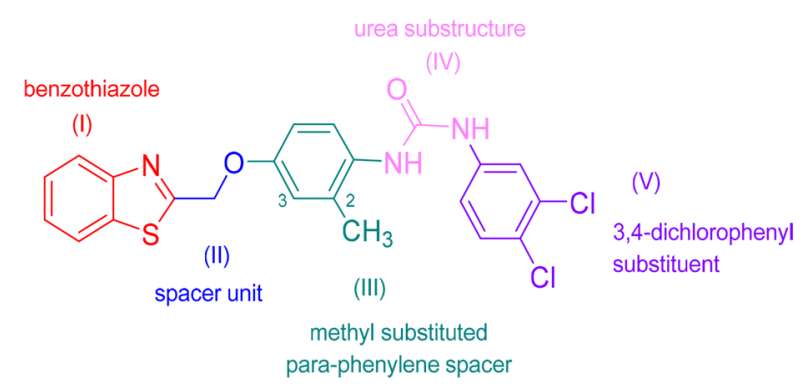

Figure 1. Structural overview of the lead compound diflapolin (1).

epoxyeicosatrienoic acids (EETs) toward the corresponding dihydroxyeicosatrienoic acids (DHETrEs).,12 In contrast to the anti-inflammatory EETs, DHETrEs possess less beneficial properties and are discussed to be rather related to inflammatory processes. Thus, $\mathrm{sEH}$ inhibition leads to increased EETs levels accompanied by advantageous effects. ${ }^{6}$ Despite intense investigations, so far no $\mathrm{sEH}$ inhibitor has reached the market, but currently, some promising candidates are undergoing clinical trials. ${ }^{13,14}$ Common to all $\mathrm{sEH}$ inhibitors is a urea or carboxamide substructure that forms hydrogen bonds with key residues within the binding site (Tyr383, Tyr466, and Asp355). ${ }^{15}$

Diflapolin also suppresses leukotriene (LT) formation by targeting the 5-LO-activating protein (FLAP). ${ }^{10,11} \mathrm{AA}$ is converted by 5-LO in a two-step reaction to $\mathrm{LTA}_{4}$ the precursor of chemotactic $\mathrm{LTB}_{4}$ and vasoactive cysteinyl LTs. ${ }^{5}$ Early FLAP inhibitors such as MK-886 and MK-591 showed promising results but were never brought to the market due to detrimental pharmakokinetics. However, novel inhibitors are currently undergoing clinical trials up to phase III. ${ }^{16}$

The increased interest is based on the fact that FLAP inhibitors are effective against chronic inflammatory diseases and show a better risk-benefit profile compared to direct 5LO inhibitors. ${ }^{17}$ The combination of sEH inhibition and the inhibition of LT biosynthesis has been shown to be beneficial as Meirer et al. reported a dual 5-LO/sEH inhibitor with an imidazo-[1,2-a]pyridine core, linked with an aryl urea moiety by a carbon chain. ${ }^{2,18}$ The target combination of FLAP and sEH is promising because inhibition of FLAP might specifically block LT formation without targeting other LOs that are related to the biosynthesis of anti-inflammatory specializedpro-resolving mediators (SPM). ${ }^{19}$

Encouraged by the promising pharmacological profile and the in vivo results of the dual sEH/FLAP inhibitor diflapolin, ${ }^{11}$ we elucidated structural essential moieties in this SAR study and aimed to improve the activity profile of diflapolin by modifying the lead compound. Molecular modeling was used to rationalize the activity data and to guide the optimization process. $\mathrm{sEH}$ inhibitors in general possess a urea or a carboxamide moiety and FLAP inhibitors frequently consist of a bicycle, so we decided to keep substructure I (benzothiazole) and substructure IV (urea) intact and focused on subunits II, III, and V (Figure 1).

To determine the effects of the substitution pattern on subunit $\mathrm{V}$ and the influence of the methyl group and its position on subunit III, compounds 11-27 were prepared using a synthetic strategy developed in our group. Treatment of 2-chloromethylbenzothiazole (2) with 3-methyl-4-nitrophenol, 2-methyl-4-nitrophenol, or 4-nitrophenol in the presence of cesium carbonate, sodium carbonate, and potassium iodide in refluxing acetone led to compounds 35. Subsequent reduction of the nitro function with hydrogen and Raney nickel as catalyst afforded the corresponding substituted aniline derivatives 7-9. Finally, the desired target compounds with ether subunit (i.e., compounds 11-27) became accessible by reaction of 7-9 with the appropriate phenyl isocyanates. In addition to the 3,4-dichlorophenyl substituted compounds, the corresponding 3,5-dichlorophenyl, the three different monochlorophenyl and derivatives with unsubstituted phenyl were prepared.

Moreover, three compounds with modified spacer unit II were prepared. Derivative $\mathbf{2 8}$, which is characterized by a formal exchange of the oxygen by sulfur was synthesized in an analogous manner as diflapolin by using 4-nitrothiophenol instead of 4-nitrophenol (Scheme 1). The derivatives with a two-carbon spacer unit (i.e., compounds 31 and 32) became accessible by reaction of 3,4-dichlorophenylisocyanate with the already known 2 2-(4-aminostryryl) and the 2-(4-aminophenylethyl) substituted benzothiazole $(\mathbf{2 9}, \mathbf{3 0})$ (Scheme 2$)$.

Scheme 2. Procedure for the Synthesis of Compounds 31 and $32^{a}$

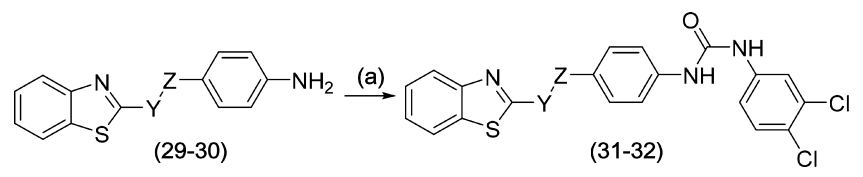

${ }^{a} \mathrm{Y}-\mathrm{Z}=\mathrm{CH}_{2}-\mathrm{CH}_{2}: \mathrm{CH}=\mathrm{CH}$. Reagents and conditions: (a) 3,4dichlorophenylisocyanate in THF, room temperature.

Scheme 1. General Procedure for the Synthesis of Compounds 3-28 ${ }^{a}$

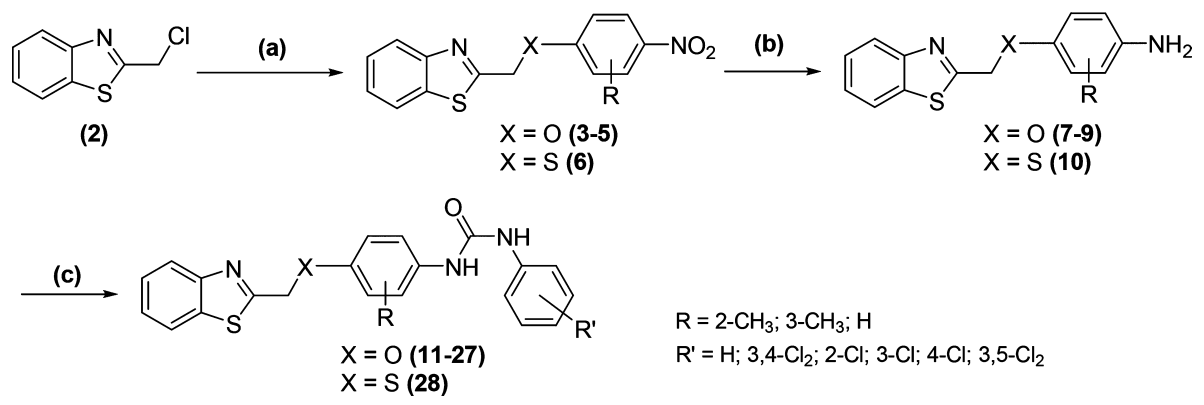

${ }^{a}$ Reagents and conditions: (a) methyl substituted or unsubstituted 4-nitrophenol or thiophenol, $\mathrm{Na}_{2} \mathrm{CO}_{3}, \mathrm{Cs}_{2} \mathrm{CO}_{3}$, $\mathrm{KI}$, acetone, reflux; (b) $\mathrm{H}_{2}$, Raney nickel in methanol; (c) corresponding phenylisocyanates in THF, room temperature. 
Table 1. Activity Data for Compounds $11-28$ and $31,32^{a}$

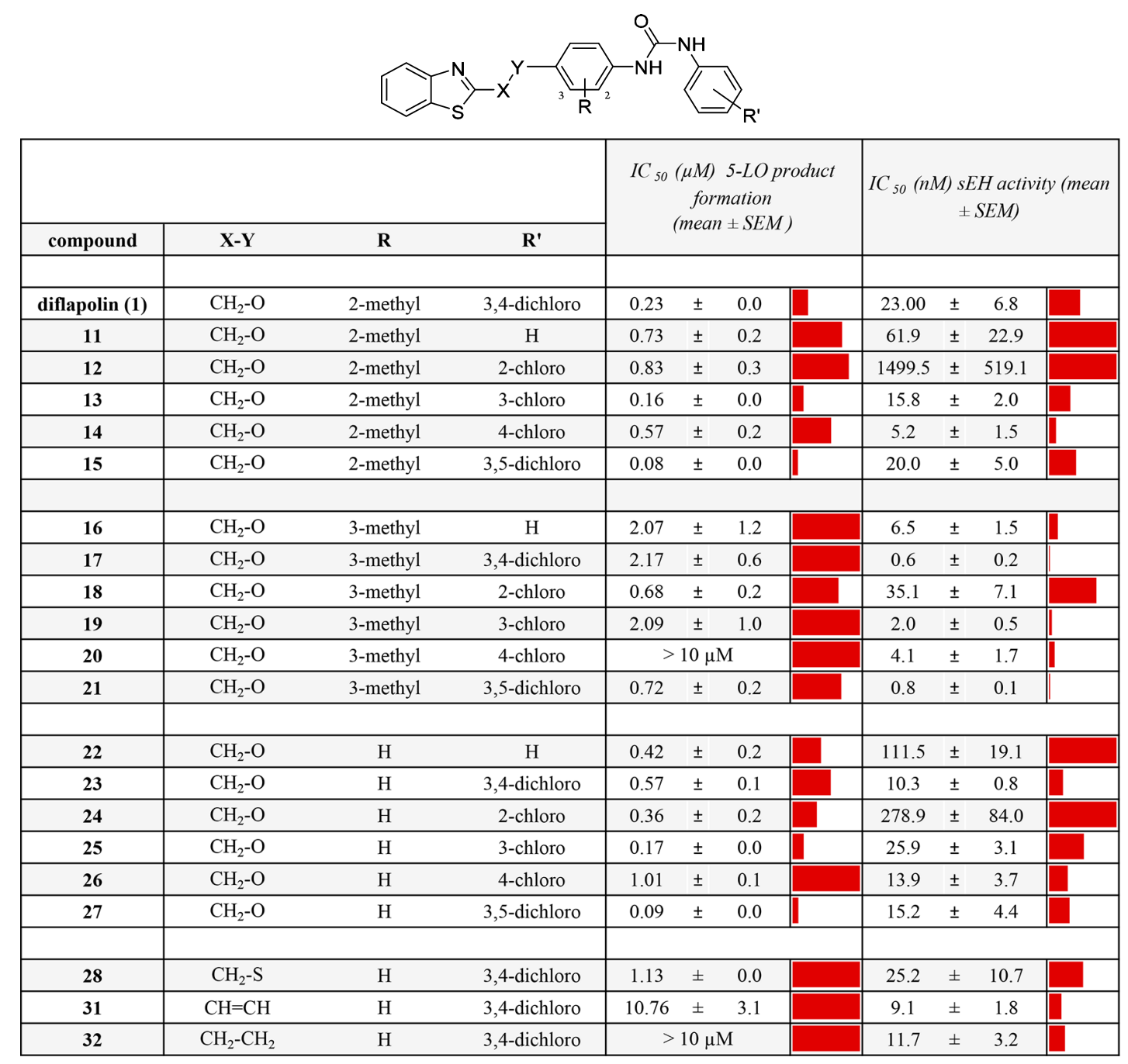

${ }^{a_{T}}$ The $0-1 \mu \mathrm{M}$ range for 5-LOX product formation and $0-50 \mathrm{nM}$ range for $\mathrm{sEH}$ activity were chosen for bar chart presentation (red) of $\mathrm{IC}_{50}$ values $(n \geq 3)$ in order to visualize the inhibitory activity of the test compounds.

All diflapolin derivatives were tested for their inhibitory potency against sEH and FLAP in biological assays (Table 1). $\mathrm{IC}_{50}$ values were determined for both assays using GraphPad Prism 8 software (Graphpad Software Inc., San Diego, CA).

The following SAR regarding subunits III and $\mathrm{V}$ was observed for FLAP-mediated 5-LO product formation in human neutrophils: Evaluation of the subunit III shows that the 3-methyl (16-21) group impairs FLAP activity compared to the derivatives with a 2-methyl group (1 and 11-15). Removing the methyl group altogether (22-27) leads to a slight increase in FLAP activity, with the exception of 26.

Within the 2-methyl substituted series, the 3,5-chloro (15) and the 3-chloro (13) modifications lead to superior inhibition than diflapolin.

In the generally less potent series of 3-methyl derivatives, the 3-chloro substituted compound $\mathbf{1 8}$ was found to be the most active. Within the generally more active series without the methyl group, also the 3,5- and the 3-chloro substitutions (25, 27) displayed the highest FLAP inhibitory activity.

Thus, we conclude that for FLAP inhibition, methyl substitution of the phenylene ring is clearly preferred at 2position but is not essential. The chlorine substitution pattern (subunit V) can be varied, but the most promising derivatives are 3-chloro and 3,5-chloro.

Regarding inhibition of sEH, compounds with a 3-methyl moiety at the phenylene ring (subunit III) are most effective, but also 2-methyl and unsubstituted phenylene lead to active compounds, and many of them are superior to diflapolin (1317, 19-21, 23, 26, 27) Notably, a chloro substituent in the ortho-position of the terminal phenyl reduces the inhibitory activity against sEH $(12,18,24)$.

Modifications at the spacer unit II had little effect on sEH but strongly affected FLAP activity. While a thioether (28) only reduced FLAP activity, compounds 31 and 32 lose their inhibitory effects against FLAP, which might be explained by the loss of the hydrogen bond acceptor.

The SARs are summarized in Figure 2.

We also conducted a Free-Wilson analysis on compounds 1 and 11-27 to quantify the influence of the specific modifications on the overall activity on the two targets (see Supporting Information).

The model showed that the 3-methyl modification on subunit III is lowering FLAP inhibitory activity, assigning a negative score of -0.923 to this modification Also, the 4- 
$-\mathrm{CH}_{2}-\mathrm{O}$ - is crucial for FLAP inhibition

$-\mathrm{CH}_{2}$-S- leads to decreased FLAP inhibition,

$-\mathrm{CH}_{2}-\mathrm{CH}_{2}$ - and $-\mathrm{CH}=\mathrm{CH}$ - is not tolerated by FLAP

$-\mathrm{CH}_{2}-\mathrm{O}-,-\mathrm{CH}_{2}-\mathrm{S}-,-\mathrm{CH}_{2}-\mathrm{CH}_{2}$ - or $-\mathrm{CH}=\mathrm{CH}$ - is tolerated by $\mathrm{sEH}$

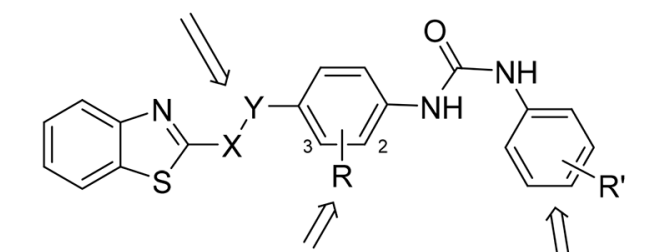

2- $\mathrm{CH}_{3}$ or $\mathrm{H}$ is favoured for FLAP inhibition

$3-\mathrm{CH}_{3}$ is preferred for $\mathrm{SEH}$ inhibition

$\mathrm{H}$ is tolerated by FLAP and $\mathrm{sEH}$

$3-\mathrm{Cl}$; $3,4-\mathrm{Cl}_{2}$, or $3,5-\mathrm{Cl}_{2}$ is preferred for FLAP inhibition $4-\mathrm{Cl}$ is not tolerated by FLAP $2-\mathrm{Cl}$ is not tolerated by $\mathrm{SEH}$

Figure 2. Summary of the SAR for FLAP and sEH inhibition (green, favorable moieties; red, unfavorable moieties).

chloro modification at subunit $\mathrm{V}$ is not generally beneficial for FLAP inhibition $(-0.404)$, while chloro substitution in the meta positions result in positive activity contributions.

Most substitutions enhanced or did not affect sEH activity; only a 2-chloro substitution on subunit $\mathrm{V}$ clearly lowered it and received a negative contribution in the Free-Wilson analysis $(-0.910)$. 3-Methyl at subunit III is preferred but not required for $\mathrm{sEH}$ inhibition.

To further investigate the SAR, the ligands were docked into the sEH binding site. Docking on FLAP is not shown because the crystal structure was not suitable for docking.

In the sEH docking simulation, modifications in compound 13 (modified substitution on subunit V) and 21 (3-methyl on subunit III) did not affect the typical binding pattern between the urea moiety and the catalytic triad of sEH, that is, Asp 355, Tyr 466, and Tyr383 (Figure 3). The least active compound

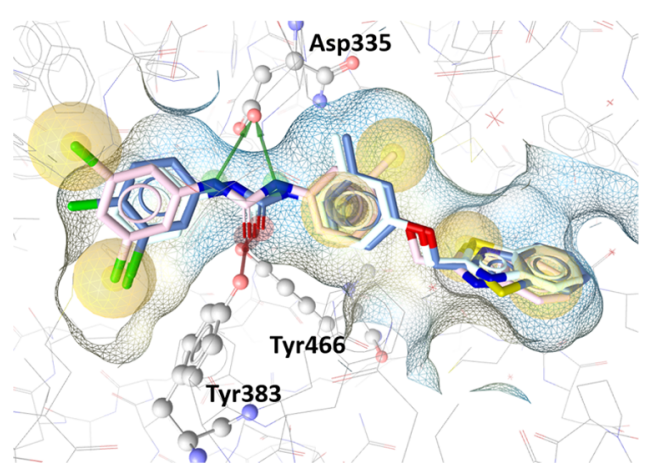

Figure 3. Diflapolin (blue) shown together with 21 (pink) and 13 (white) in sEH. The simulation indicates that the binding mode is not affected by the modifications and that all form the key interactions with the catalytic triad of sEH, that is, Asp355, Tyr466, and Tyr383. Yellow spheres represent hydrophobic contacts, green arrows represent hydrogen bond donors, and red arrows mark hydrogen bond acceptors.

24, with the chlorine in ortho-position, was docked with a turn of the urea moiety, which prohibited the hydrogen bonding with the catalytic site (Figure 4), explaining the loss of activity.

In conclusion, our SAR study of novel diflapolin derivatives revealed important information regarding relevant substructures to target FLAP and $\mathrm{sEH}$, respectively. While both activities can be improved on one of the targets, it is challenging to modify the molecule and optimize both

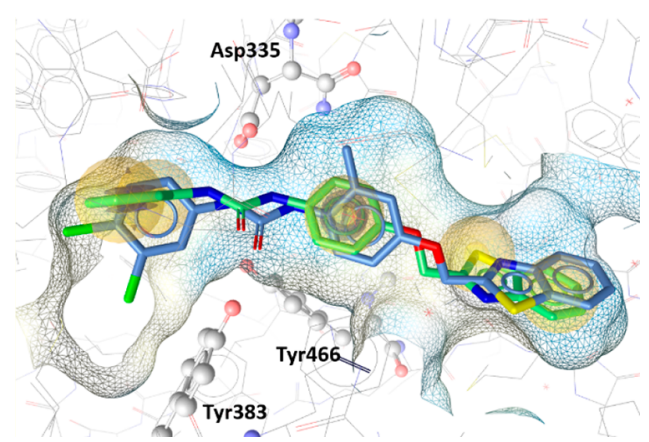

Figure 4. Diflapolin is shown in blue and inactive compound 24 in green. The hydrogen bond interactions with $\mathrm{sEH}$, indicated in Figure 3 , are only predicted for diflapolin. Because of the turn caused by the steric hindrance of the chlorine in the ortho-position in compound $\mathbf{2 4}$, the urea moiety is no longer forming the key interactions.

activities at the same time. For compounds 15 and 27, both activities were increased.

\section{ASSOCIATED CONTENT}

\section{Supporting Information}

The Supporting Information is available free of charge on the ACS Publications website at DOI: 10.1021/acsmedchemlett.8b00415.

Synthetic procedures, spectral data and elemental analysis for key compounds, computational methods, Free-Wilson analysis, biological assay protocols (PDF)

\section{AUTHOR INFORMATION}

\section{Corresponding Authors}

*For B.M.: phone, +43 512 507-58262; fax, +43 512 50758299; E-mail, Barbara.matuszczak@uibk.ac.at.

*For U.G.: phone, +49 3641 949-811; fax, +49 3641 949-802; E-mail, Ulrike.garscha@uni-jena.de.

\section{ORCID}

Daniela Schuster: 0000-0002-9933-8938

Barbara Matuszczak: 0000-0002-4507-8739

Present Addresses

${ }^{\#}$ L.V., M.S., D.S., B.M.: Institute of Pharmacy, Department of Pharmaceutical Chemistry, Center for Chemistry and Biomedicine, University of Innsbruck, Innrain 80-82, A-6020 Innsbruck, Austria.

${ }^{\nabla}$ E.R., C.K., A.T., J.F., U.G., O.W.: Chair of Pharmaceutical/ Medicinal Chemistry, Friedrich-Schiller University Jena, Philosophenweg 14, D-07743 Jena, Germany.

OS.S., V.T.: Institute of Pharmacy, Department of Pharmacognosy, Center for Chemistry and Biomedicine, University of Innsbruck, Innrain 80-82, A-6020 Innsbruck, Austria.

D.S.: Institute of Pharmacy, Department of Pharmaceutical and Medicinal Chemistry, Paracelsus Medical University Salzburg, Strubergasse 21, A-5020 Salzburg, Austria.

${ }^{\mathbb{I}}$ V.H.-O.: Fraunhofer Institute for Molecular Biology and Applied Ecology IME, Branch for Translational Medicine and Pharmacology TMP, Theodor-Stern-Kai 7, 60596 Frankfurt am Main, Germany.

\section{Author Contributions}

The manuscript was written through contributions of all authors and all authors have given approval to the final version of the manuscript. 


\section{Notes}

The authors declare no competing financial interest.

\section{ACKNOWLEDGMENTS}

We thank the German Research Council (SFB1278 Polytarget, to O.W., and GA 2101/2-1, to U.G.) for financial support. L.V. and M.S. acknowledge the support by the University of Innsbruck ("Doktoratsstipendium der Nachwuchsförderung"). V.T. is funded by the Austrian Science Fund (FWF): project T942-B30.

\section{ABBREVIATIONS}

AA, arachidonic acid; BSA, bovine serum albumin; COX, cyclooxygenase; cPLA2, cytosolic phospholipase A2; CYP, cytochrome; DHETrEs, dihydroxyeicosatrienoic acids; DMLs, designed multiple ligands; DMSO, dimethyl sulfoxide; EETs, epoxyeicosatrienoic acids; FLAP, 5-LO-activating protein; LO, lipoxygenase; LT, leukotriene; PHOME, 3-phenyl-cyano(6methoxy-2-naphthalenyl)methylester-2-oxiraneacetic acid; SAR, structure-activity relationships; $s E H$, soluble epoxide hydrolase; SPM, specialized-pro-resolving mediators; THF, tetrahydrofuran.

\section{REFERENCES}

(1) Bennett, M.; Gilroy, D. W. Lipid mediators in inflammation. Microbiol. Spectr. 2016, 4, 1-21.

(2) Meirer, K.; Steinhilber, D.; Proschak, E. Inhibitors of the arachidonic acid cascade: Interfering with multiple pathways. Basic Clin. Pharmacol. Toxicol. 2014, 114, 83-91.

(3) Waltenberger, B.; Garscha, U.; Temml, V.; Liers, J.; Werz, O.; Schuster, D.; Stuppner, H. Discovery of Potent Soluble Epoxide Hydrolase (sEH) Inhibitors by Pharmacophore-Based Virtual Screening. J. Chem. Inf. Model. 2016, 56, 747-62.

(4) Dinarello, C. A. Anti-inflammatory Agents: Present and Future. Cell 2010, 140, 935-50.

(5) Temml, V. Dissertation: Molecular modeling and computational investigation of targets within the inflammatory cascade. Ph.D. Thesis. Universität Innsbruck 2014.

(6) Koeberle, A.; Siemoneit, U.; Buehring, U.; Northoff, H.; Laufer, S.; Albrecht, W.; Werz, O. Licofelone suppresses prostaglandin E2 formation by interference with the inducible microsomal prostaglandin E2 synthase-1. J. Pharmacol. Exp. Ther. 2008, 326, 975-982.

(7) Morphy, R.; Rankovic, Z. Designing multiple ligands - medicinal chemistry strategies and challenges. Curr. Pharm. Des. 2009, 15, 587600.

(8) Morphy, R.; Rankovic, Z. Designed multiple ligands. An emerging drug discovery paradigm. J. Med. Chem. 2005, 48, 6523-43.

(9) Fischer, L.; Hornig, M.; Pergola, C.; Meindl, N.; Franke, L.; Tanrikulu, Y.; Dodt, G.; Schneider, G.; Steinhilber, D.; Werz, O. The molecular mechanism of the inhibition by licofelone of the biosynthesis of 5-lipoxygenase products. Br. J. Pharmacol. 2007, 152, 471-80.

(10) Temml, V.; Garscha, U.; Romp, E.; Schubert, G.; Gerstmeier, J.; Kutil, Z.; Matuszczak, B.; Waltenberger, B.; Stuppner, H.; Werz, O.; Schuster, D. Discovery of the first dual inhibitor of the 5lipoxygenase-activating protein and soluble epoxide hydrolase using pharmacophore-based virtual screening. Sci. Rep. 2017, 7, 42751.

(11) Garscha, U.; Romp, E.; Pace, S.; Rossi, A.; Temml, V.; Schuster, D.; Konig, S.; Gerstmeier, J.; Liening, S.; Werner, M.; Atze, H.; Wittmann, S.; Weinigel, C.; Rummler, S.; Scriba, G. K.; Sautebin, L.; Werz, O. Pharmacological profile and efficiency in vivo of diflapolin, the first dual inhibitor of 5-lipoxygenase-activating protein and soluble epoxide hydrolase. Sci. Rep. 2017, 7, 9398.

(12) Morisseau, C.; Hammock, B. D. Epoxide hydrolases: mechanisms, inhibitor designs, and biological roles. Annu. Rev. Pharmacol. Toxicol. 2005, 45, 311-33.
(13) Lazaar, A. L.; Yang, L.; Boardley, R. L.; Goyal, N. S.; Robertson, J.; Baldwin, S. J.; Newby, D. E.; Wilkinson, I. B.; Tal-Singer, R.; Mayer, R. J.; Cheriyan, J. Pharmacokinetics, pharmacodynamics and adverse event profile of GSK2256294, a novel soluble epoxide hydrolase inhibitor. Br. J. Clin. Pharmacol. 2016, 81, 971-9.

(14) Guedes, A.; Galuppo, L.; Hood, D.; Hwang, S. H.; Morisseau, C.; Hammock, B. D. Soluble epoxide hydrolase activity and pharmacologic inhibition in horses with chronic severe laminitis. Equine. Vet. J. 2017, 49, 345-351.

(15) Eldrup, A. B.; Soleymanzadeh, F.; Farrow, N. A.; Kukulka, A.; De Lombaert, S. Optimization of piperidyl-ureas as inhibitors of soluble epoxide hydrolase. Bioorg. Med. Chem. Lett. 2010, 20, 571575 .

(16) Werz, O.; Gerstmeier, J.; Garscha, U. Novel leukotriene biosynthesis inhibitors $(2012-2016)$ as anti-inflammatory agents. Expert Opin. Ther. Pat. 2017, 27, 607-620.

(17) Pettersen, D.; Davidsson, O.; Whatling, C. Recent advances for FLAP inhibitors. Bioorg. Med. Chem. Lett. 2015, 25, 2607-12.

(18) Meirer, K.; Roedl, C. B.; Wisniewska, J. M.; George, S.; Haefner, A.-K.; Buscato, E. 1.; Klingler, F.-M.; Hahn, S.; Berressem, D.; Wittmann, S. K.; Steinhilber, D.; Hofmann, B.; Proschak, E. Synthesis and structure-activity relationship studies of novel dual inhibitors of soluble epoxide hydrolase and 5-lipoxygenase. J. Med. Chem. 2013, 56, 1777-1781.

(19) Serhan, C. N.; Petasis, N. A. Resolvins and Protectins in Inflammation Resolution. Chem. Rev. (Washington, DC, U. S.) 2011, $111,5922-5943$.

(20) Becker, R.; Theobald, H.; Schirmer, U.; Spiegler, W.; Seufert, W.; Wuerzer, B. Urea derivatives and their use for controlling undesired plant growth. German Patent DE3148291A1, 1983. 